\title{
Cloud Networking Computing
}

\author{
Jonghun $\mathrm{Kim}^{1} \cdot$ Hector John T. Manaligod ${ }^{2} \cdot$ Jieun Lee $^{3} \cdot$ Sunmoon Jo ${ }^{4}$
}

Published online: 18 February 2019

(c) Springer Science+Business Media, LLC, part of Springer Nature 2019

Recently, the research on human-centered information communications that can be utilized and accessed anytime, anywhere in the cloud network has been actively conducted. Cloud network computing is comprised of of hypervisor server virtualization, router-based network virtualization, distributed file processing, file service platforms, and right management. Red Hat's Ceph Storage, IBM's OCIS, the GlusterFS scalable network filesystem, the DataDirect Networks Web Object Scaler, and Hitachi Vantara's Hitachi Content Platform have been developed as distributed storage that provides large-scaled storage space in the network. In addition, cloud integration monitoring is inviting attention as a paradigm by combining cloud computing technology and the fate, management, and mirroring of a route. Although there are many advantages, such as reliability, efficiency, scalability, interoperability and security, research should be done to solve matters such as data integration, response time, and maintenance. This theme issue introduces selected research papers and includes trends in topics like distributed data management, hybrid networking systems, hybrid vulnerability analysis, interactive mobile applications, time-series pattern mining, power measurement, cloud transmission, peer-to-peer network, blockchain network, hybrid wireless protocols, cryptography communication protocols, the mobile cloud network, multi-channel protocols, and priority-based communications.

The first paper by Jeong et al. [1] proposes a probabilistic approach based on the Basic Local Alignment Search Tool (BLAST) to ensure high accuracy and improve the search

Jonghun Kim

ddcome11@daum.net

Hector John T. Manaligod

hjmanaligod@gmail.com

Jieun Lee

jieun.lee.0072@gmail.com

Sunmoon Jo

sunmoon@pcu.ac.kr

1 Department of Software Convergence Engineering, Inha University, 100 Inha-ro, Michuhol-gu, Incheon 22212, South Korea

2 School of Management and Information Technology, Computer Applications Department, De La Salle College of St. Benilde, Manila, Philippines

3 Korea Convergence Society, 65, Buldang 21-ro, Seobuk-gu, Cheonan-si, Chungcheongnam-do, South Korea

4 Department of Computer Information Technology Education, Paichai University, 155-40 Baejae-ro, Seo-gu, Daejeon 35345, South Korea 
speed of high-performance bioinformatics. This study focuses on accessibility by efficiently processing large-scale bioinformatics and biomedical engineering with heuristic factors to access large-capacity bioinformatics information. It allocates bioinformatics information by classifying the property hierarchical groups with sequence analysis and linkage similarity. The paper by Kim et al. [2] develops an enhanced order-based genetic algorithm to overcome the shortest-path problem with precedence constraints of higher complexities. The suggested algorithm aims to solve precedence-constrained and unconstrained mazetype shortest-path problems in a maze-type sparse network. This study flexibly develops a constructive order-based genetic algorithm and a fitness-switching genetic algorithm with precedence constraints, fitness switching, and fitness leveling.

The paper by Park et al. [3] presents a hybrid vulnerability management for self-risk analysis using a risk evaluation computing. The aim of this proposed technique is to evaluate self-risks and to improve accuracy through intelligent vulnerability detection. It uses hybrid dynamic and static analysis to solve weak points at each stage and improve the accuracy of vulnerable self-risk detection. The paper by Lim et al. [4] develops SmartSenior: automatic content personalization through assessment of an intelligent user interface/user experience (UI/UX) and semi-supervised learning. This study proposes automatic adaptations, a cognitive model, a behavior model, and UI/UX modules to help the user by taking into account both feedback and behavioral data. It also facilitates quality of life (QoL) and social connectivity by making dynamic personalized computing devices from interface aspects to suit the cognitive needs of the individual. The paper by Kim et al. [5] suggests a time-series life pattern mining using big life-log for a health cloud computing. This study aims to generate sleep patterns using a sequential pattern and context-based user clustering to improve QoL. In addition, it explores time-series mining patterns and cloud networking computing using an expectation maximization (EM) algorithm. Sequence pattern mining contributes to improving physical healthcare computing and increasing individual accessibility in the cloud computing.

The paper by Park et al. [6] introduces high-speed remote power measurement using analog and digital measurement computing. It aims to find the feasibility and communication relationship between high-speed remote measurement, peak-based power measurement, and root mean square-based power measurement. In addition, it uses a measurement frequency range to determine characteristics by assessing the validity of high-frequency resonance in the cloud computing. The paper by Yi et al. [7] presents semantic representation computing for communications between wireless robots and humans as an egocentric-semantic topology. This study focuses on semantic map building and the human navigation-inspired representation that a wireless robot uses in egocentric place recognition, dead reckoning, and reorientation in robot mapping and localization. In addition, it uses the human inspired representation of spatial relations and landmarks to signify robot mapping, and uses localization networking for adaptive navigation indoors. The paper by Han et al. [8] presents spatial image retrieval computing using content-based image retrieval (CBIR) with factor analysis expressing the features. The proposal focuses on a multi-direction Gabor filter expressing segmented features for multi-scale edge computing. It extracts quantized color correlation and the contour direction of the features using chromatic distribution and a quantized hue, saturation, and intensity value (HSV) histogram algorithm for relative similarity and specific object computing.

The paper by Lee et al. [9] develops a deep-packet-inspection-based convergence solution for security vulnerabilities in industrial communications networking. This study is on security industry control networking computing though self-similarity, an industrial protocol gateway, and unidirectional communications to improve the multi-channelized 
security level of information and personalized communications networking. The paper by Kim et al. [10] suggests priority-based transmission using k-means clustering in widearea communications with mobile edge computing. This study groups devices using k-means clustering according to priority-based data transmission and traffic characteristics. Classified end devices determine wireless channel access time to avoid collisions and improve transmission efficiency in low-power wide-area networks (LPWANs) with long delays. The paper by Chung et al. [11] suggests a blockchain-based topic encapsulation mining process for cognitive manufacturing and monitoring. The proposed method focuses on manufacturing context information and human-body motion sensing with ambient intelligence (AmI) computing devices using side chain-based data block-consensus blockchain networking in distributed computing. In addition, this is a consortium blockchain-distributed ledger using adaptive feature extraction and a topic encapsulation mining process in peer-to-peer blockchain networking computing.

The paper by Jin et al. [12] introduces a secure communications protocol using a ring learning with error (RLWE)-based fully homomorphic encryption process in cloud-centric Internet of Things networking. The study focuses on an encryption communications protocol using message management and authentication. In addition, a communications protocol consists of a key procedure, registration, and management to prevent privacy threats and information leakage. It uses a homomorphic encryption algorithm, hash tree-based certificate management, and re-encryption and decryption processes after performing authentication between the user and the gateway in cloud networking. The paper by Ahn et al. [13] presents panoramic image generation using an ambient intelligence device with a camera for the cloud-computing environment. The proposed method aims to distribute the high computational load using segmented images transmitted from the AmI devices and by computing homography error information. It uses feature extraction and feature descriptor generation to improve computational complexity and for high quality in cloud networking computing. The paper by Cho et al. [14] suggests $360^{\circ}$ video traffic reduction using mobile streaming in cloud computing. This study focuses on field of view (FoV): high quality for FoV and background frame low quality for non-FoV. In addition, it uses a front on the backward frame (FBF) to reduce network load without degrading quality of experience (QoE), based on streaming with appropriate HTTP quality, by making high-resolution video of the FoV variable and getting traffic gain.

The paper by Kim et al. [15] develops a health bigdata based interactive growth management system in wireless personalized communications that focuses on children and adolescents to solve obesity issues and unbalanced growth. They develop an IEEE 1107320601 communications protocol between ubiquitous computing devices (wearable, mobile, AmI devices, etc.) as an inter-gateway and an outer-gateway, with biometric sensors and a communications security protocol for ambient intelligence (AmI) computing. The paper by Lee et al. [16] proposes a sequence pattern-mining process for wireless personalized communications stream data to detect disappearing and emerging patterns. This study focuses on a sliding-window computing and frequent-event time-series pattern mining for batchfree event in wireless personalized communications. The last paper by Kim et al. [17] suggests associative feature extraction using the Apriori Algorithm from data relations in a large health data set. This study aims to extract features from documents obtained through health cloud computing, providing a knowledge base in wireless personalized communications. It uses association-mining analysis between keywords and a weighted core health corpus from huge transactions. In addition, it applies a term frequency-inverse document frequency of the hash, title, and emphasis tag for improving the accuracy. 
We really appreciate all the authors and the reviewers for their valuable contributions. We would also like to express thanks to Editors-in-Chief Professor Ramjee Prasad and the members of the journal.

\section{References}

1. Jeong, Y. S., \& Shin, S. S. (2018). Probabilistic approach processing scheme based on BLAST for improving search speed of bioinformatics. Wireless Personal Communications. https://doi. org/10.1007/s11277-018-5955-3.

2. Kim, J. W., \& Kim, S. K. (2018). Genetic algorithms for solving shortest path problem in maze-type network with precedence constraints. Wireless Personal Communications. https://doi.org/10.1007/ s11277-018-5740-3.

3. Park, J. P., Choo, Y. S., \& Lee, J. H. (2018). A hybrid vulnerability analysis tool using a risk evaluation technique. Wireless Personal Communications. https://doi.org/10.1007/s11277-018-5959-z.

4. Lim, K., Hooshyar, D., Ji, H., Lee, S., \& Jo, J. (2018). SmartSenior: Automatic content personalization through semi-supervised learning. Wireless Personal Communications. https://doi.org/10.1007/ s11277-018-5947-3.

5. Kim, J. C., \& Chung, K. (2018). Mining based time-series sleeping pattern analysis for life big-data. Wireless Personal Communications. https://doi.org/10.1007/s11277-018-5983-z.

6. Park, S. J., Lim, S. K., \& Park, S. M. (2018). High-speed remote power measurement by communication of the maximum and minimum measurement value. Wireless Personal Communications. https:// doi.org/10.1007/s11277-018-6082-X.

7. Yi, C., Cho, J., \& Suh, I. H. (2018). Semantic representation for communication between human and wireless robot. Wireless Personal Communications. https://doi.org/10.1007/s11277-018-5949-1.

8. Han, H. H., Ryu, S. H., Chae, G. S., \& Lee, S. H. (2018). Image retrieval using CBIR including light position analysis. Wireless Personal Communications. https://doi.org/10.1007/s11277-018-5943-7.

9. Lee, J. K., Lee, S. W., \& Lee, K. H. (2018). An implementation of convergence security solution for overcoming of security vulnerabilities in industrial control communication network. Wireless Personal Communications. https://doi.org/10.1007/s11277-018-5970-4.

10. Kim, D. Y., \& Kim, S. (2018). Data transmission using K-means clustering in low power wide area networks with mobile edge cloud. Wireless Personal Communications. https://doi. org/10.1007/s11277-018-5742-1.

11. Chung, K., Yoo, H., Choe, D., \& Jung, H. (2018). Blockchain network based topic mining process for cognitive manufacturing. Wireless Personal Communications. https://doi.org/10.1007/s11277-018-5979-8.

12. Jin, B. W., Park, J. O., \& Mun, H. J. (2018). A design of secure communication protocol using RLWEbased homomorphic encryption in IoT convergence cloud environment. Wireless Personal Communications. https://doi.org/10.1007/s11277-018-6083-9.

13. Ahn, H., Lee, J. H., \& Cho, H. J. (2018). Research of panoramic image generation using IoT device with camera for cloud computing environment. Wireless Personal Communications. https://oi. org/10.1007/s11277-018-5972-2.

14. Cho, S., Kim, D., Kim, C., Jeong, K. Y., \& Sohn, C. B. (2018). 360-degree video traffic reduction using cloud streaming in mobile. Wireless Personal Communications. https://doi.org/10.1007/s11277-018-5984-y.

15. Kim, J., Jang, H., Kim, J. T., Pan, H. J., \& Park, R. C. (2018). Big-data based real-time interactive growth management system in wireless communications. Wireless Personal Communications. https:// doi.org/10.1007/s11277-018-5978-9.

16. Lee, K. M., Han, C. S., Nam Jun, J., Lee, J. H., \& Lee, S. H. (2018). Batch-free Event sequence pattern mining for communication stream data with instant and persistent events. Wireless Personal Communications. https://doi.org/10.1007/s11277-018-5985-x.

17. Kim, J. C., \& Chung, K. (2018). Associative feature information extraction using text mining from health big data. Wireless Personal Communications. https://doi.org/10.1007/s11277-018-5722-5.

Publisher's Note Springer Nature remains neutral with regard to jurisdictional claims in published maps and institutional affiliations. 

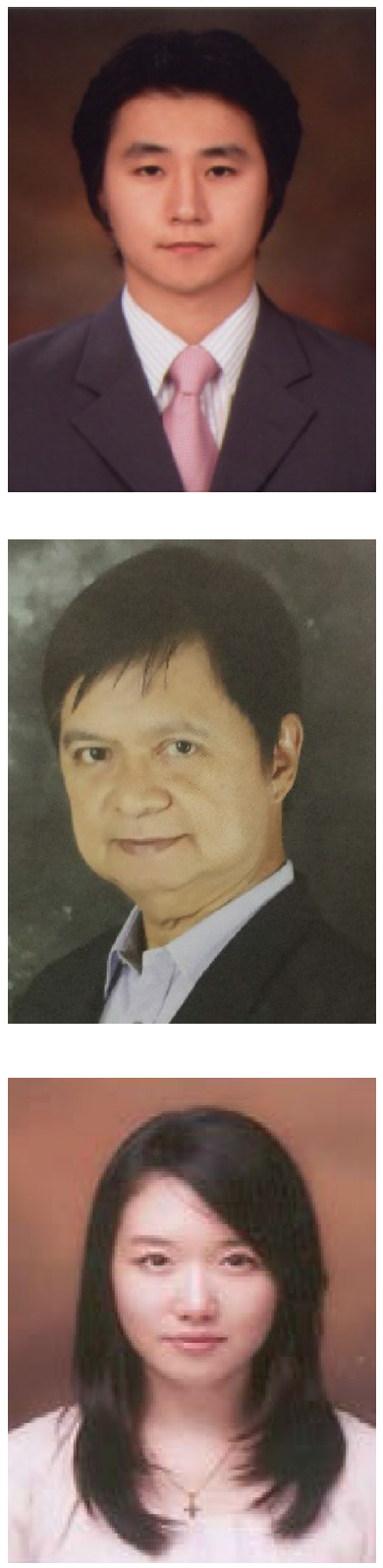

Jonghun Kim received Ph.D. degree in Computer Science \& Information Engineering from Inha University, South Korea. From 2013 to 2018, he was a professor in the Department of Computer Software, Daelim University, South Korea. Since 2019, he is a professor in Department of Software Convergence Engineering, Inha University, South Korea. His research interests include Cloud Networking Computing, Cloud Transmission, Peer-to-Peer Networking, Blockchain Networking, Recommendation, Hybrid Wireless Protocols, and Multimedia Convergence. He serves as Executive Editing Director of International Conference Convergence Technology, as Steering Committees of the International Conference on Digital Policy \& Management. Also, he is editorial board members of several International Journals.

Hector John T. Manaligod is a professor at the De La Salle-College of Saint Benilde in the School of Management and Information Technology. He took his doctoral degree under the Erasmus Mundus program at the Universidade de Santiago De Compostela, Spain where he graduated as cum laude. He graduated at the University of the Philippines with specialization on Measurement and Evaluation. He also taught computer, research, statistics courses at the University of the Philippines, Ateneo University, AMA Computer University, among others. $\mathrm{He}$ is a member of the Chief Information Forum of the Philippines and Philippine Society of Information Technology Educators. He wrote researches and designed/programmed various softwares. His research interests include artificial intelligence, neural network, data mining, business analytics, and Python languages. He looks forward to publishing his own books on Business Intelligence and Analytics, Data Management, Advanced Statistics. His advocacy is to raise awareness and use of information technology to the grassroot level.

Jieun Lee is the executive secretary of Korea convergence Society, South Korea. She supervised and managed conferences including International Conference Convergence Technology, International Conference on Internet of Thing and Convergence and International Research Conference on Innovation, Technology and Sustainability. Her research topics are Sensibility Design, IT Convergence, Digital Computing, Bigdata Mining, Hybrid Networking Systems, Hybrid P2P Protocols, Graphics, and Digital Content. Furthermore, she has controlled special issues of prestigious international journals. Also, she is editorial board members of several International Journals. 


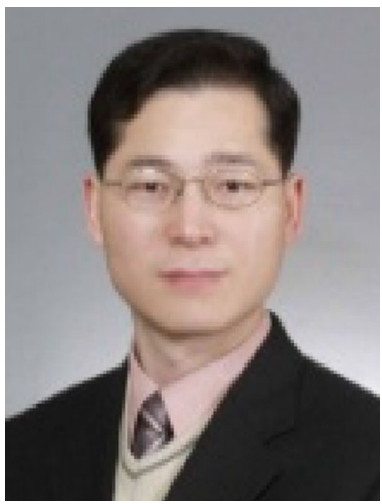

Sunmoon Jo is a professor in the Department of Computer Information Technology Education, Paichai University, South Korea. His research interests include telecommunication, XML, security, knowledge system, deep learning, hybrid P2P, and wireless communication. He serves as Director and a member of the Editorial Committee of the Korea Knowledge Information Technology Society, as Director of the Korea Contents Association, as Member of the Korean Society for Internet Information, as Member of the Korea Information Processing Society, as Member of the Institute of Electrical and Electronics Engineers, as Steering Committees of International Conference on Digital Policy \& Management, and as Excellent Professor Award of the August 2015 Paichai University President. 